\title{
基于 Android 系统的制丝生产实时监视 APP 的开发
}

\section{Development of Real-time Monitoring APP for Silk Production Based on Android System 贺欣 王新亭 陈飞云}

Xin He Xinting Wang Feiyun Chen

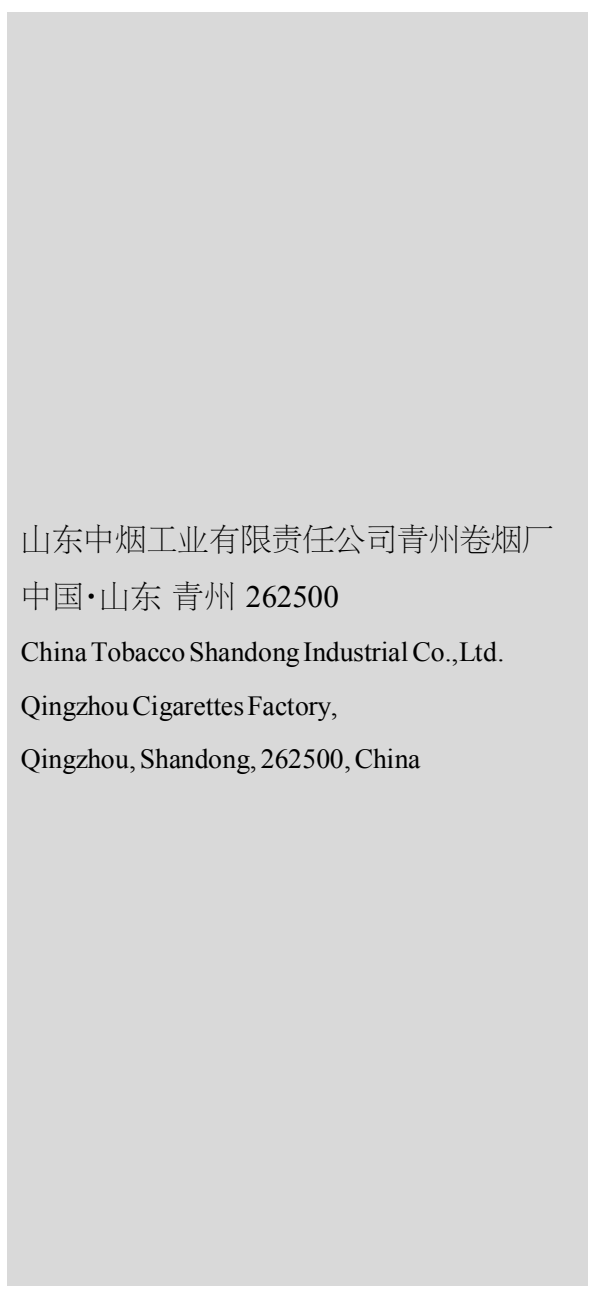

【摘要】在卷烟厂制丝车间生产过程中, 管理人员需实时掌握各工艺段的生产情况, 以便 及时知晓各工艺段生产信息并合理安排生产任务。目前, 制丝生产的原始数据可通过计算 机终端查询, 亟需开发一款基于 Android 系统的 APP 将生产数据在手机终端实时展示出 来。APP 的数据来源为 MES 系统和制丝中控系统, 前台手机端通过用户点击向后台服务 端发出一条 HTTP 请求，服务端收到请求之后从 MES 系统和制丝中控系统获取所需数 据, 并以 JSON 格式返回给手机端, 然后手机端再对此 JSON 格式的数据进行解析和处理, 最后展示到手机界面上。APP 不仅能够根据用户选择实时展示相关生产信息,也验证了将 MES 系统和制丝中控系统中的数据实时显示在手机 APP 界面上的技术可行性。

【Abstract】In the production process of cigarette factory's silk-making workshop, managers need to grasp the production situation of each process section in real time so as to know the production information of each process section in time and arrange the production tasks reasonably. At present, the raw data of silk production can be queried by computer terminal. It is urgent to develop an APP based on Android system to display the production data in real time at mobile terminal. The data source of APP is MES system and silk-making central control system. The front-end mobile phone sends an HTTP request to the back-end server through the user's click. After receiving the request, the server obtains the required data from MES system and silk-making central control system, and returns it to the mobile phone in JSON format. Then the mobile phone side parses and processes the data in JSON format, and finally displays it to the mobile phone interface. The APP can not only display relevant production information in real time according to the user's choice, but also verify the technical feasibility of real-time display of data from MES system and silk manufacturing central control system on the mobile phone APP interface.

【关键词】制丝生产; 实时监视; 技术; 系统

【Keywords】silk production; real-time monitoring; technology; system 【DOI】10.36012/etr.v1i4.716

\section{1 引言}

卷烟厂的制丝车间是典型的流水线生产，所以实时掌握 各工艺段的生产情况，对知晓各工艺段生产信息及合理安排 生产任务等方面十分有必要。目前, 中国青州卷烟厂了解制丝 生产情况主要有以下两种途径:一是到生产现场查看, 此方式 只能了解当前生产批次, 不能直观地了解当天的生产进度; 二 是在台式电脑通过 MES 或制丝中控等系统查看, 此方式不够 便捷, 并且 MES 和制丝中控系统中的数据量较大, 数据的针 对性较弱 ${ }^{[1]}$
随着工业互联网时代的到来, 手机 APP 在生活和日常工 作中被越来越广泛地应用, 同时, 基于 Android 系统开发的手 机 APP 在手机应用程序中所占的比例越来越大。在此背景 下, 开发一款基于 Android 系统的制丝生产专用的 APP, 实现 从 MES 系统和制丝中控系统中实时提取数据并直观地展示 在手机界面上, 方便工作人员及时了解生产信息的技术条件 已经成熟。

\section{2 解决的技术问题}

制丝生产实时监视 APP 的菜单形式效仿微信的底部菜 
单样式, 主要分为两部分内容: 储柜信息和生产计划 ${ }^{[2]}$ 。储柜 信息展示制丝车间所有储柜名称, 可根据用户选择, 进一步展 示所选储柜各单柜的状态、在存牌号、批次号、文本号、进料开 始时间、进料结束时间、出料开始时间、实时存量。储柜状态分 为待料、进料、出料、存料 4 种状态, 并且以不同颜色显示状态 文字。生产计划展示制丝线主要工艺段 (预混处理段、一次加 料处理段、烘丝处理段、掺配加香处理段)当天的生产计划, 可 根据用户选择,进一步展示所选工艺段当天的工单运行情况。 工单运行情况按列表样式显示, 包括运行状态、生产牌号、文 本号。工单运行状态如果是“完成”, 则用户可以通过点击该工 单, 进而显示“工单完成时间”; 工单运行状态如果是“运行”, 则用户可以通过点击该工单, 进而显示“工单开始时间”。通过 此 APP 的展示, 可以使用户直观且全面地了解制丝线生产运 行情况, 并能根据展示内容合理安排生产进度。

\section{3 具体的技术方案}

目前, 开发 Android 版手机 APP 的主流工具有两种 ${ }^{[3]}$ : eclipse 和 Android Studio, 鉴于前台和后台开发环境的统一, 此次开发选择 eclipse 软件。前台手机端界面布局采用 XML 语言编写, 逻辑控制采用 Java 语言; 界面设计力求简洁直观, 易于操作。由于手机内存有限, 不能从前台直接读取数据库, 需要建立后台服务, 完成从数据库中提取数据并传送给手机 端的工作, 后台服务端同样采用 Java 语言编写。

本款 APP 的数据来源为 MES 系统和制丝中控系统, 具 体的数据包括预混柜、咜叶柜、保温柜、混丝柜、悾梗柜及梗 丝柜的各个单柜的状态、在存牌号、批次号、文本号、进料开 始时间、进料结束时间、出料开始时间、实时存量, 预混处理 段、新预混处理段、一次加料处理段、新一次加料处理段、气 流烘丝处理段、薄板烘丝 4800 处理段及掺配加香处理段的 当天任务的工单状态、生产牌号、文本号、工单开始时间、工 单结束时间。

数据在手机端展示的具体过程为: 前台手机端通过用户 点击向后台服务端发出一条 HTTP 请求, 服务端收到请求之 后从 MES 系统和制丝中控系统获取所需数据, 并以 JSON 格 式返回给手机端, 然后手机端再对此 JSON 格式的数据进行 解析和处理, 最后展示到手机界面上。制丝生产实时监视 APP 技术路线如图 1 所示。

\section{APP 实施的效果}

制丝生产实时监视 APP 借助智能手机, 向制丝车间管理 人员和生产人员直观地展示制丝线的生产信息, 不仅方便管

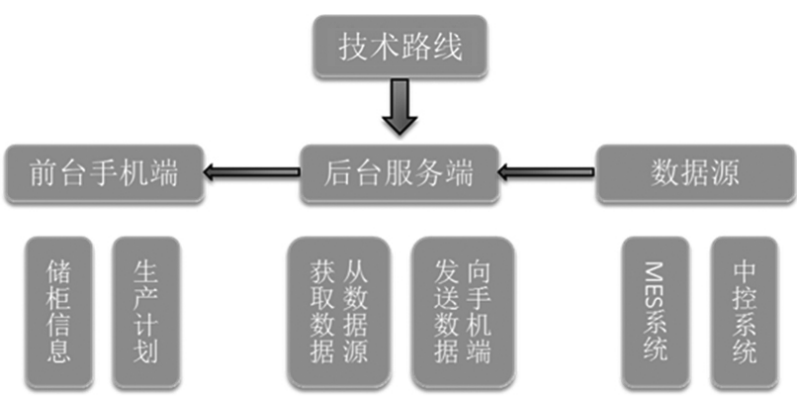

图 1 制丝生产实时监视 APP 技术路线

理人员和生产人员了解各工艺段在产情况, 也能让管理人员 更及时合理地安排生产进度, 提高了工作效率, 节省了时间成 本。与在电脑终端查看 MES 或制丝中控系统的数据信息相比, 此 APP 能根据制丝车间日常生产情况，有针对性地从 MES 系统和制丝中控系统中提取小部分数据, 使用户更快速、更直 观地查看所需信息,保证了程序的运行速度。

在储柜信息模块,应用程序按储柜状态 (分为待料、进料、 出料、存料 4 种状态) 以不同颜色显示, 用户就能更直接明了地 看到各储柜储存状态, 进而根据需要查看某一状态下的储柜存 储信息。状态为进料或出料的储柜, 实时存量可以让用户知晓 当前储柜存量, 便于用户对下步生产工作采取对策。由进料结 束时间和出料开始时间可以得知当前储柜内的烟叶 (丝) 实际 储存时长, 进而判断是否符合工艺标准要求的储存时长, 使判 断烟叶(丝)的储存时长是否达到标准的方式更为简单直观。

在生产计划模块, 将制丝线主要工艺段统一呈现, 方便车 间管理人员全面了解各工艺段生产进度, 有利于把握排产节 奏, 进一步提升各工艺段协同生产能力。各工艺段的生产计划 展示剔除了制丝中控系统提供的不必要的信息, 只保留用户 需要知晓的信息,使手机界面一目了然,信息针对性更强。

\section{5 结语}

此 APP 的开发过程, 打通了将 MES 系统和制丝中控系 统中的数据实时显示在手机 APP 界面上的技术路径, 为今后 开发 MES 系统、SPC 系统、底层制造系统等生产系统的移动 平台, 或将智能移动端与企业现有生产经营系统进行整合奠 定了基础。

\section{参考文献}

[1]赵亮,张维. 基于 Android 技术的界面设计与研究 [D]. 徐州: 徐 州建筑职业技术学院,2001.

[2]陈璟, 陈平华, 李文亮.Android 内核分析[J]. 现代计算机(专业 版),2009(11):112-115.

[3]韩超.Android 经典应用程序开发 $[\mathrm{M}]$. 北京: 电子工业出版社, 2012. 